Ann. Biol. anim. Bioch. Biophys., 1977, 17 (1), 21-31.

\title{
Les vitesses de renouvellement des ARN du foie de carpe (Cyprinus carpio L.) soumise à un jeûne total et prolongé
}

\author{
par G. BOUCHE, Y. CREACH ef A. SERFATY \\ avec la collaboration technique de M. DASSAIN
}

Laboratoire d'Ecophysiologie animale

Université Paul-Sabatier, 38, rue des 36-Ponts

31000 Toulouse, France

Summary. Turnover rate of hepatic RNA in carp (Cyprinus carpio L.) after prolonged total starvation.

1. Turnover rate and half-life of hepatic RNA in fed or starved carp are determined after a single intraperitoneal injection of uridine- ${ }^{3} \mathrm{H}-5$.

2. Acute starvation causes a decrease in the turnover time of total nuclear and ribosomal RNA in carp liver.

3. Fractional catabolic and synthesis rates remain high in severely starved fish, showing that constant fraction RNA, present after prolonged total starvation, has a high metabolic activity.

4. Augmentation of the cytoplasmic RNA turnover rate is correlated with increase of the exportation rate of nuclear RNA into the cytoplasmic liver cells.

\section{Introduction.}

Le contenu en ARN d'une cellule esi la résultante de deux processus antagonistes, la synthèse ef la dégradation, qui peuvent être indépendamment altérés par différents facteurs comme le jeûne protéique ou total (Enwonwu, Stambaugh et Sreenby, 1971).

Les poissons peuvent résister à de longues périodes de jeûne naturel (Fontaine et Olivereau, 1963 ; Love, 1970 ; Sorvachev, 1957) ou expérimental (Love, 1970 ; Creach, 1972). Cependant, nous ne possédons aucune donnée sur l'influence du jeûne sur le turn-over des différentes fractions ribonucléiques des tissus de ces Vertébrés inférieurs. Nous avons retenu comme matériel d'étude le tissu hépatique de la carpe dont le métabolisme ribonucléique est profondément affecté par l'état de dénutrition. En effet, le jeûne total et prolongé provoque dans le foie de la carpe commune une chute importante de la teneur en $A R N$ total et en $A R N$ ribosomal $\left(A R N_{r}\right)$ liée à une baisse du taux de polysomes (Bouche, Creach et Gas, 1970 ; Bouche, Narbonne et Serfaty, 1972 ; Bouche, Narbonne et Creach, 1972). Par ailleurs, la persistance, dans les derniers mois d'inanition, d'une fraction constante d'ARN $N_{r}$ et de polysomes pose le problème de savoir si cet $A R N$ « résiduel » conserve une certaine activité métabolique. 
Ces recherches complètent celles obtenues précédemment sur le renouvellement des protéines hépatiques, plasmatiques et musculaires (Bouche et Vellas, 1975).

\section{Matériel et méthodes.}

Des carpes communes d'un poids initial compris entre 150 et $200 \mathrm{~g}$ sont mises à jeûner en décembre dans les conditions précédemment décrites (Bouche et Vellas, 1975). Un lot de témoins est régulièrement nourri avec de l'aliment complef pour carpes contenant 37 p. 100 de protéines et 4 p. 100 de lipides. La croissance de ces animaux témoins est de l'ordre de 11 p. 100 par mois pour la durée de l'expérimentation.

Quatre individus sont sacrifiés tous les mois afin de suivre l'évolution de l'ARN total hépatique. Lorsque les pertes en ARN total sont de l'ordre de 20 p. 100 (3 mois de jeûne) quarante carpes à jeun reçoivent une injection intrapéritonéale d'Uridine${ }^{3} \mathrm{H}-5$ (C.E.A. Saclay-France) à raison de $100 \mu \mathrm{Ci}$ pour $100 \mathrm{~g}$ de poids vif. Le reste des individus est laissé à jeun et est utilisé lorsque le taux d'ARN total hépatique représente 30 à 35 p. 100 de la valeur initiale * (7 mois de jeûne). A ce moment, les jeûneurs et un nombre équivalent de carpes régulièrement alimentées ( 30 individus) reçoivent une injection d'Uridine- ${ }^{3} \mathrm{H}-5$ à la même dose que celle donnée ci-dessus.

Les sujets, prélevés au hasard, sont ensuite sacrifiés à des temps variables après l'injection du précurseur radioactif (tabl. 1).

TABLEAU 1

Temps d'abattage après l'injection d'Uridine- ${ }^{3} \mathrm{H}-5$

\begin{tabular}{cccc}
\hline \multicolumn{1}{c}{ Temps } & Nombre d'animaux & Temps & Nombre d'animaux \\
\hline $1 \mathrm{~h}$ & $4 \mathrm{~J}_{7} ; 4 \mathrm{~T}$ & $144 \mathrm{~h}(6$ jours $)$ & $4 \mathrm{~J}_{3}$ \\
$6 \mathrm{~h}$ & $4 \mathrm{~J}_{3} ; 4 \mathrm{~J}_{7} ; 4 \mathrm{~T}$ & $168 \mathrm{~h}(7$ jours) & $4 \mathrm{~J}_{7} ; 4 \mathrm{~T}$ \\
$24 \mathrm{~h}(1$ jour) & $4 \mathrm{~J}_{3} ; 4 \mathrm{~J}_{7} ; 4 \mathrm{~T}$ & $192 \mathrm{~h}(8$ jours) & $4 \mathrm{~J}_{3}$ \\
$48 \mathrm{~h}$ (2 jours) & $4 \mathrm{~J}_{3}$ & $240 \mathrm{~h}(10$ jours $)$ & $4 \mathrm{~J}_{7} ; 4 \mathrm{~T}$ \\
$74 \mathrm{~h}$ (3 jours) & $4 \mathrm{~J}_{7} ; 4 \mathrm{~T}$ & $336 \mathrm{~h}(14$ jours) & \\
$96 \mathrm{~h}$ (4 jours) & $4 \mathrm{~J}_{3}$ & & \\
\hline
\end{tabular}

$J_{3}$ : Carpes à jeun sacrifiées après 3 mois d'inanition totale au mois de janvier.

$\mathbf{J}_{7}$ : Carpes à jeun sacrifiées après 7 mois d'inanition tolale au mois de mai.

$\mathrm{T}$ : Carpes régulièrement nourries sacrifiées en même temps que les ieûneurs $J_{7}$.

\section{Fractionnement cellulaire.}

Toutes les opérations de fractionnement cellulaire sont réalisées en chambre froide à la température de $0-4{ }^{\circ} \mathrm{C}$.

* Remarque : Ces pertes en ARN total (65 à 70 p. 100) correspondent aux pertes maximales observées chez les carpes dénutries. L'ARN « résiduel » représente alors la fraction constante qui subsiste dans les derniers mois d'inanition. Par contre, dans les premiers mois de jeûne, notre expérimentation intéresse la fraction labile de l'ARN qui disparaît lors du jeûne (Bouche, Creach et Gas, 1970). 
a) Isolement des noyaux.

Des échantillons de $0,5 \mathrm{~g}$ de foie sont repris par $6 \mathrm{ml}$ de milieu Tris- $\mathrm{HCl}$

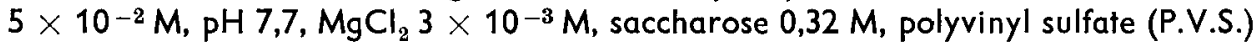
$20 \mu \mathrm{g} / \mathrm{ml}$ ef homogénéisés une minute au moyen d'un homogénéiseur Ultra-Turrax tournant à basse vitesse. La suspension ainsi obtenue est centrifugée $10 \mathrm{mn}$ à $700 \mathrm{~g}$. Chaque homogénat est contrôlé au microscope après coloration au lugol ef la vifesse d'homogénéisation est adaptée en fonction de ce contrôle.

Le culot de noyaux est lavé en le mettant en suspension dans une solution de saccharose $0,25 \mathrm{M}$ contenant du Tris- $\mathrm{HCl} 5 \times 10^{-2} \mathrm{M}, \mathrm{pH} 7,7$, du $\mathrm{MgCl}_{2} 3 \times 10^{-3} \mathrm{M}$, ef du P.V.S. $20 \mu \mathrm{g} / \mathrm{ml}$, et à nouveau centrifugé $10 \mathrm{mn}$ à $700 \mathrm{~g}$.

b) Isolement des ribosomes totaux, extractions et dosages des ARN et de l'ADN.

Les ribosomes tolaux sont isolés selon une technique précédemment décrite (Bouche, Narbonne et Serfaty, 1972). L'ARN nucléaire et l'ARN $\mathrm{r}_{r}$ sont extraits et dosés par les méthodes décrites par Bouche (1975).

L'ARN total du foie est extrait selon la technique de Schmidt ef Thannhauser (1945) ef dosé par absorption directe à $260 \mathrm{~nm}$ ef par la réaction à l'orcinol (Moule, 1953). L'ADN total hépatique est extrait selon la méthode de Vannemaker, Banks et Wunner (1965) ef dosé par absorption directe à 260 et $290 \mathrm{~nm}$ et par la réaction à la diphénylamine (Burton, 1956).

\section{Détermination de la radioactivité.}

Des fractions des divers hydrolysats ribonucléiques $(0,2 \mathrm{ml})$ sont pipetées dans des fioles de comptage ; $1 \mathrm{ml}$ de solvène et $10 \mathrm{ml}$ de mélange scintillant $(5 \mathrm{~g}$ PPO-100 $\mathrm{mg}$ POPOP q.s.p. $1 \mathrm{I}$ de toluène) sont ajoutés. La détermination de la radioactivité est réalisée à l'aide du compteur à scintillation liquide Intertechnique SL 30.

\section{Analyse des résultats.}

La décroissance de la radioactivité des ARN isolés peut être représentée d'après Campbell et Kosterlitz (1952) ef Loeb, Howell et Tomkins (1965) par une courbe exponentielle d’équation :

$$
A_{t}=A_{0} e^{-k t}
$$

où $A_{0}$ est la radioactivité maximale au temps $t_{0}$, $A_{t}$ la radioactivité au temps $t$. La représentation semi-logarithmique de l'équation (1) est une droite dont l'équation $\log A=\log A_{0}-k t$ est calculée par la méthode des moindres carrés. Le taux fractionnel catabolique (T.F.C.) est défini comme le pourcentage du pool total d'ARN dégradé (Jarnum ef Jansen, 1972). Il est égal à $k \times 100$. La demi-vie apparente $(\uparrow 1 / 2)$ des ARN considérés correspond à $\frac{\log 2}{\mathrm{k}}$, le temps de renouvellement à $\frac{1}{\mathrm{k}}$.

Le test de comparaison des coefficients de régression des droites obtenues par la méthode des moindres carrés est utilisé pour différencier statistiquement la pente de ces droites. 


\section{Résultats.}

L'expression de la radioactivité des acides ribonucléiques en $\mu \mathrm{Ci} \times 10^{-4} / \mathrm{mg}$ ADN nous permet de tenir compte de la taille du pool d'ARN considéré et de son évolution lors du jeûne (Blobel et Potter, 1968).

I. ARN tofal (ARN).

L'ARN total hépatique des carpes régulièrement nourries est rapidement marqué (fig. 1a). La radioactivité de l'ARN atteint $320 \pm 120 \mu \mathrm{Ci} \times 10^{-4} / \mathrm{mg}$ ADN

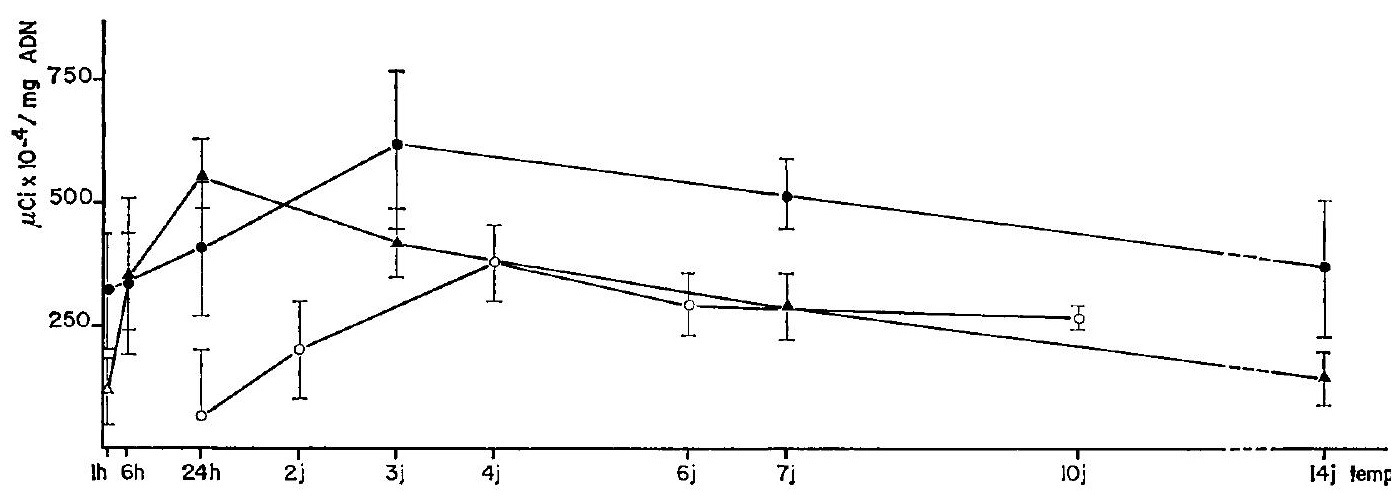

Chaque point des courbes correspond à la moyenne obtenue sur quatre individus $\left(\bar{x} \pm t_{0,05} \times S \mathrm{~m}\right)$. $\longrightarrow$ témoins, $\mathrm{O} \longrightarrow \mathrm{O}$ jeûneurs, $(3$ mois) $\Delta \longrightarrow \mathbf{\Lambda}$ jeûneurs $(7$ mois $)$.

FIG. 10. - Effet du jeâne sur le marquage (exprimé en $\mu \mathrm{Ci} \times 10^{-4} / \mathrm{mg}$ ADN) de l'ARN total hépatique à divers temps après l'injection intrapéritonéale d'Uridine- ${ }^{3} \mathrm{H}-5$

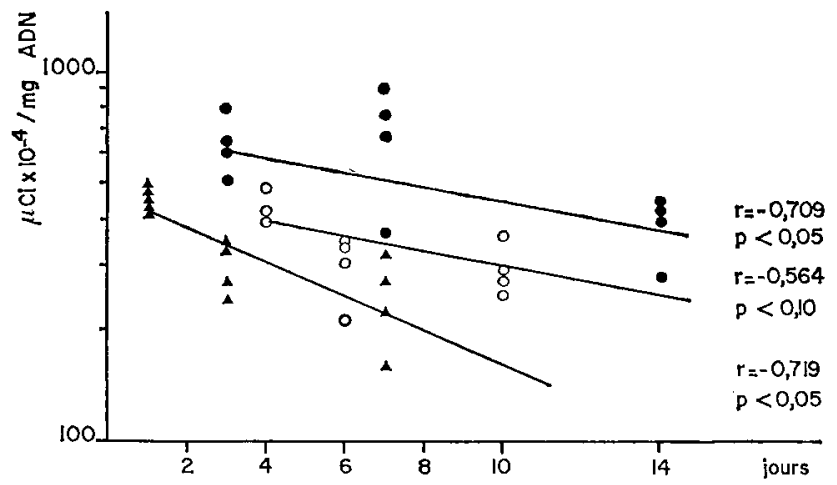

- - témoins, $\mathrm{O}-\mathrm{O}$ jeûneurs (3 mois), $\boldsymbol{\Delta}-\boldsymbol{\Delta}$ jeûneurs $(7$ mois) $r=$ coefficient de corrélation

$P=$ sevil de signification de $r$ et de l'hypothèse de linéarité (tesi de Fischer).

FIG. 1b. - Représentation semi-logarithmique de la décroissance de la radioactivité de l'ARN total hépatique des carpes à jeun ou régulièrement nourries 
une heure après l'injection d'Uridine- ${ }^{3} \mathrm{H}-5$. Par la suite, l'augmentation de la radioactivité est progressive pour atteindre une valeur maximale, au $3^{e}$ jour.

La représentation semi-logarithmique de la décroissance de la radioactivité de I'ARN des carpes nourries (fig. $1 b$ ) montre que les pertes de radioactivité s'effectuent lentement ( $k=0,040$, tabl. 2). Le taux fractionnel de catabolisme (T.F.C.) est de 4 p. 100. Le gain journalier en ARN de ces carpes est de 0,15 p. 100 (tabl. 2) ; il s'ensuit que le taux de synthèse est de 4,15 p. 100. La demi-vie et le temps de renouvellement de l'ARN des carpes nourries sont respectivement de 15,4 et de 22,17 jours (tabl. 2).

\section{TABLEAU 2}

Demi-vies, temps de renouvellement, taux fractionnel de catabolisme ef équation de régression de la représenfation semi-logarithmique de la décroissance de la radioactivité des ARN du foie des carpes à jeun ou régulièrement alimentées

\begin{tabular}{|c|c|c|c|c|c|}
\hline & $\begin{array}{l}\text { Demi-vie } \\
(+1 / 2) \\
\text { en jours }\end{array}$ & $\begin{array}{c}\text { Temps de } \\
\text { renou- } \\
\text { vellement } \\
\text { en jours }\end{array}$ & $\begin{array}{c}\text { Taux fractionnel } \\
\text { catabolisme } \\
\text { journalier } \\
\text { (T.F.C.) en p. } 100\end{array}$ & $\begin{array}{c}\log A=\log A_{0}-k t \\
A-A_{0} \text { en } \mu C i \times 10^{-4} / m g A D N\end{array}$ & $\begin{array}{l}\text { Variations } \\
\text { journalières } \\
\text { du pool d'ARN } \\
\text { en p. } 100\end{array}$ \\
\hline ARN $\begin{array}{l}\mathrm{T} \\
\mathrm{j}_{3} \ldots \\
\mathrm{J}_{7} \ldots\end{array}$ & $\begin{array}{l}15,4 \\
10,34 \\
7,21 \\
\end{array}$ & $\begin{array}{l}22,17 \\
14,83 \\
10,36\end{array}$ & $\begin{array}{l}4,0 \\
6,7 \\
9,6\end{array}$ & $\begin{array}{l}\log A=\log 706,1-0,040 \\
\log A=\log 503,1-0,067 \\
\log A=\log 444,8-0,096 t\end{array}$ & $\begin{array}{l}+0,15 \\
-0,23 \\
0\end{array}$ \\
\hline 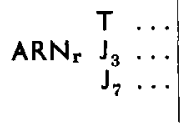 & $\begin{array}{l}7,07 \\
4,81 \\
5,4\end{array}$ & $\begin{array}{c}10,18 \\
6,93 \\
7,8\end{array}$ & $\begin{array}{r}9,8 \\
14,4 \\
12,8\end{array}$ & $\begin{array}{l}\log A=\log 842,04-0,098 t \\
\log A=\log 417,9-0,144 t \\
\log A=\log 1176-0,128 t\end{array}$ & $\begin{array}{c}+0,32 \\
-0,22 \\
0\end{array}$ \\
\hline
\end{tabular}

T : carpes régulièrement nourries sacrifiées en mai.

$\mathrm{J}_{3}$ : carpes à jeun depuis 3 mois sacrifiées en janvier.

$J_{7}$ : carpes à jeun depuis 7 mois sacrifiées en mai.

Le marquage de l'ARN du foie des carpes à jeun s'effectue progressivement. Cependant, l'augmentation de la radioactivité de l'ARN des individus à jeun depuis 7 mois est plus rapide que celle des sujets en inanition totale depuis 3 mois (fig. $1 a$ ).

La décroissance de la radioactivité de I'ARN hépatique des jeûneurs est plus rapide que celle de I'ARN des carpes régulièrement alimentées (fig. 1b). Le jeûne entraîne une diminution du temps de renouvellement et de la demi-vie de I'ARN du foie (tabl. 2). Après 3 mois d'inanition, le T.F.C. est de 6,7 p. 100.

Sept mois de jeûne amènent le T.F.C. à 9,6 p. 100. Les pertes quotidiennes en ARN sont, à ce moment-là, insignifiantes (tabl. 2). Le taux fractionnel synthétique est alors très proche du T.F.C.

\section{ARN nucléaire $\left(A R N_{n}\right)$.}

La radioactivité de l'ARN ${ }_{n}$, exprimée en $\mu \mathrm{Ci} \times 10^{-4} / \mathrm{mg} A D N$, des noyaux d'hépatocyte de carpes à jeun est plus élevée, que celle de l'ARN $n$ du foie de carpes régulièrement nourries (fig. $2 a$ ). 
Quarante-huit heures après l'injection du précurseur radioactif, les radioactivités des $A R N_{n}$ des foies de carpes à jeun depuis 3 mois et des $A R N_{n}$ des foies de carpes à jeun depuis 7 mois ont respectivement diminué de 62 p. 100 et de 49,5 p. 100.
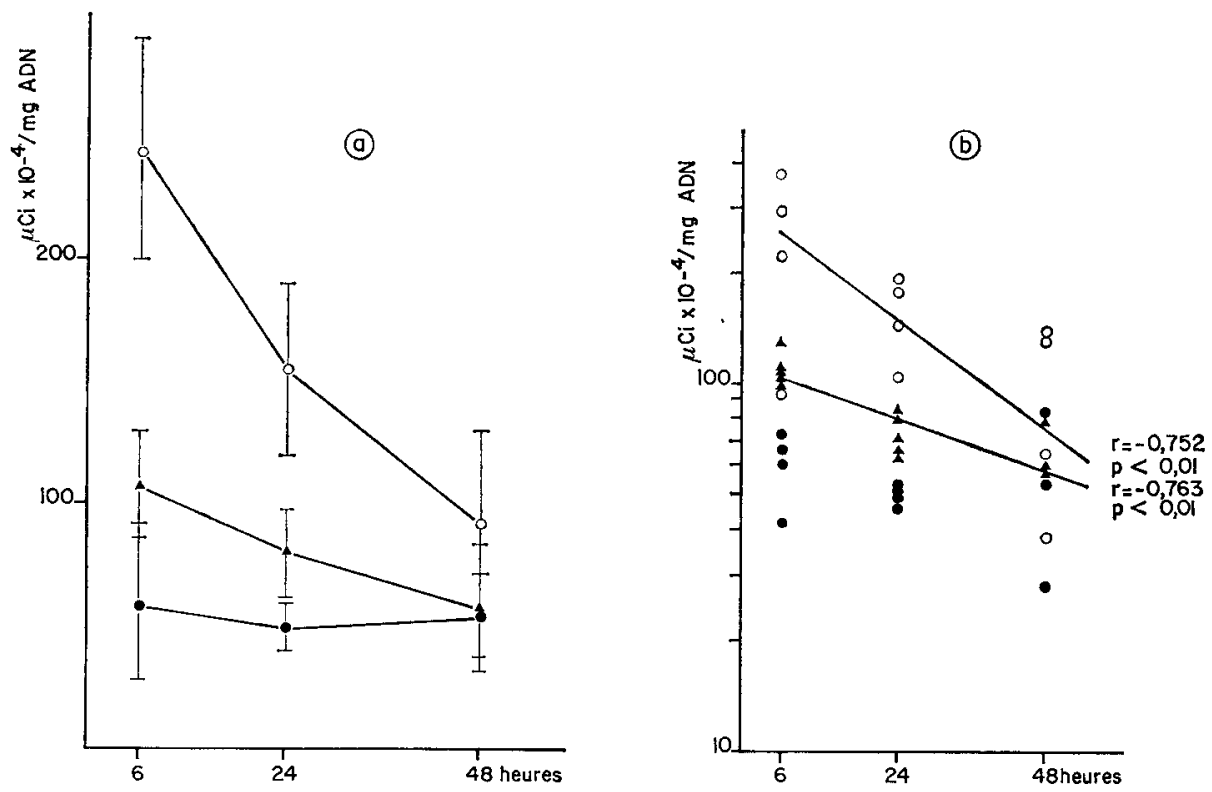

Chaque point des courbes correspond à la moyenne obtenue sur quatre poissons $\left(x \pm t_{0,05} \times S_{m}\right)$ - témoins, $\bigcirc \longrightarrow$ jeûneurs ( 3 mois), $\quad \Delta-\Delta$ jeûneurs $(7$ mois) $r=$ coefficient de corrélation

$P=$ sevil de signification de $r$ ef de l'hypothèse de linéarité (test de Fischer).

FIG. 2a. - Influence du jeûne sur la décroissance de la radioactivité de l'ARN nucléaire hépatique à divers temps après l'injection du précurseur radioactif
FIG. 2b. - Décroissance de la radioactivité de l'ARN ${ }_{n}$, représentée en coordonnées semilogarithmiques, chez les carpes à jeun

La constante de disparition de la radioactivité de l'ARN $N_{n}(k)$ dans le noyau des carpes à jeun depuis 3 mois est égal à 0,040 alors qu'elle n'est que de 0,013 chez les individus en inanition depuis 7 mois (fig. $2 b$ ).

Chez les carpes régulièrement alimentées, nous n'avons pu mettre en évidence une diminution de l'ARN ${ }_{n}$, deux jours après l'administration de l'Uridine- ${ }^{3} \mathrm{H}-5$ (fig. $2 a$ ). Nous n'avons pu déterminer chez ces carpes la constante de disparition de l'ARN $N_{n}$.

III. ARN ribosomal ef polysomal $\left(A R N_{r}\right)$.

La figure 3a représente l'évolution de la radioactivité de l'ARN $N_{r}$ des carpes à jeun ou régulièrement alimentées. Seule la diminution de cefte radioactivité $a$ été envisagée, à divers temps, après l'injection du précurseur radioactif. 


\section{Discussion.}

Les temps de renouvellement des acides ribonucléiques du tissu hépatique de la carpe que nous avons déterminés ne représentent qu'une estimation de l'activité ribonucléosynthétique du foie en raison des particularités anatomiques de cet organe (Bouche et Vellas, 1975).

D'une manière générale, les temps de renouvellement des ARN totaux et des $A R N_{r}$ des foies de Carpes régulièrement alimentées sont plus longs que ceux trouvés chez les Mammifères. Cette différence entre la carpe ef les Mammifères se retrouve également lorsque l'on détermine le turn-over des protéines hépatiques (Bouche ef Vellas, 1975). Elle peut être attribuée à la lenteur relative des processus métaboliques chez les Poissons. Chez les Mammifères, exception faite des faibles valeurs données par Hadjoliov (1966), les temps de renouvellement des mêmes ARN sont respectivement de 7,6 et 7,2 jours (Gerber, Gerber et Altman, 1960). Les demi-vies des ribosomes libres et fixés au réticulum de l'hépatocyte de rat sont également évaluées à 6,23 et 6,14 jours (Mishra, Wheldrake et Feltham, 1972).

La valeur des taux fractionnels de catabolisme de I'ARN ef de I'ARN $N_{r}$ du foie de carpe est environ le quart de celle trouvée, pour les ARN correspondants du tissu hépatique de rat, par Enwonwu, Stambaugh et Sreenby (1971). Ces auteurs notent une corrélation étroite entre les demi-vies de I'ARN et de I'ARN microsomal. II n'en est pas de même chez la carpe où la demi-vie de l'ARN est toujours supérieure à celle de l'ARN ${ }_{\mathrm{r}}$ quel que soit l'état nutritionnel de ce poisson (tabl. 2).

L'évolution de la radioactivité de l'ARN $N_{n}$ à divers temps après l'injection du précurseur ne ressemble pas, chez la carpe nourrie, à celle décrite dans le noyau du foie de rat par Blobel et Potter (1968). En effet, chez ce mammifère, la courbe de décroissance de la radioactivité de l'ARN $n$ a un aspect biphasique. La première partie de la courbe, à forte pente, représente le transport de l'ARN du noyau vers le cytoplasme de la cellule. La seconde partie, à faible pente, correspond à la dégradation de l'ARN intranucléaire. Nous retrouvons cet aspect biphasique seulement chez les jeûneurs. Nous nous sommes intéressés, dans notre travail, à la première partie de la courbe traduisant l'intensité des échanges noyau $\rightarrow$ cytoplasme. Chez la carpe régulièrement alimentée, l'exportation de l'ARN ${ }_{n}$ vers le cytoplasme semble s'effectuer très lentement.

Le jeûne total et provoqué entraîne une augmentation de la vitesse de renouvellement de l'ARN et de l'ARN $r$ de l'hépatocyte. Un jeûne de courte durée (3 mois) entraîne une augmentation du taux fractionnel de catabolisme et une diminution relative du taux fractionnel de synthèse de l'ARN. II s'ensuit que le foie perd de l'ARN. Ces pertes en ARN correspondent à la disparition de la fraction labile d'ARN (Bouche et al. 1970).

Par contre, les conséquences de la diminution du temps de renouvellement observée après 7 mois de jeûne ne sont pas les mêmes que celles décriłes précédemment. Lorsque les pertes globales en ARN sont de l'ordre de 65 p. 100, l'ARN qui subsiste dans le foie représente la fraction constante (Bouche et al. 1970). Si le taux fractionnel de catabolisme se trouvait encore augmenté, l'absence de pertes en ARN traduirait une augmentation correspondante du taux fractionnel de synthèse. 
Le maintien à des niveaux élevés des taux fractionnels de catabolisme et de synthèse, après un jeûne de longue durée, montre que l'ARN de la fraction constante a une activité métabolique élevée. On peuł penser que, comme dans le foie et le rein de rat en inanition protéique (Busch,Weill, Ledig et Mandel, 1958), cet ARN constant à renouvellement rapide est masqué par la présence d'un autre ARN à «turn-over» lent qui disparaî́ lors du jeûne.

Les modifications de renouvellement de l'ARN $N_{r}$ ne sont pas analogues à celles observées au niveau de l'ARN. En effet, si 3 mois de jeûne provoquent une diminution de la demi-vie de l'ARN, 7 mois d'inanition totale ramènent la durée de la demi-vie de cet $A R N$ à une valeur légèrement supérieure à celle enregistrée après un jeûne de courte durée (3 mois) (tabl. 2).

II semble que l'inanition totale et prolongée de la carpe comme le jeûne total ou protéique des Mammifères (Hirsch et Hiatt, 1966, Enwonwu ef Munro, 1970) puisse être divisée en 2 phases en fonction de sa durée.

La première où se produisent les pertes en ARN du foie associées à une intense diminution de la fraction polysomale (Bouche ef al. 1972) est caractérisée par une accélération de la dégradation de cet ARN. Pendant cette phase initiale, le taux fractionnel de synthèse, bien que supérieur à celui rencontré chez les carpes régulièrement alimentées est inférieur au taux fractionnel de catabolisme de l'ARN $\mathrm{r}_{\mathrm{r}}$ du foie des jeûneurs. L'augmentation des synthèses de l'ARN hépatique dans les premiers słades de jeûne a été également observée par Weber, Singhal ef Srivastava (1965) qui notent, chez les rats à jeun depuis un jour, un accroissement de 151 p. 100 de l'incorporation de l'orotate radioactif.

La seconde phase correspond à une diminution relative du faux fractionnel de catabolisme qui demeure toutefois légèrement supérieur à celui de l'ARN $N_{r}$ du foie des carpes nourries. De plus, le taux d'ARN $r$ garde une valeur constante et une population de polysomes subsiste dans l'hépatocyle pendant cette seconde partie du jeûne (Bouche et al. 1972), population polysomale qui reste hautement fonctionnelle (Bouche ef Vellas, 1975).

Le maintien d'un taux fractionnel de synthèse proche de celui trouvé chez les carpes régulièrement alimentées ne peut s'expliquer que par l'éventualité d'un recyclage intense des nucléotides libérés lors de la dégradation de l'ARN $N_{r}$. En effet, pendant cette période, le pool des nucléosides tri-phosphates libres diminue considérablement de taille (Bouche, 1975).

Les résultats obtenus au nivequ de l'ARN $\mathrm{N}_{\mathrm{n}}$ corroborent les observations faites au sujet de l'augmentation du renouvellement de l'ARN $N_{r}$ du foie de carpe lors du jeûne. En effet, l'accroissement du taux fractionnel de catabolisme de l'ARN $\mathrm{N}_{\mathrm{r}}$, lors de la première partie du jeûne, est concomittant d'une accélération des échanges noyau $\rightarrow$ cytoplasme. La décroissance de la radioactivité de l'ARN ${ }_{n}$ est alors très rapide ; la constante de pertes de radioactivité $(k)$ est égale à 0,040 .

Pendant la seconde partie du jeûne ( $7^{\mathrm{e}}$ mois), la diminution relative du taux fractionnel de catabolisme est synchrone d'un ralentissement de l'exportation de l'ARN $_{n}$ vers le cytoplasme ; la constante de pertes de radioactivité de l'ARN ${ }_{n}$ est alors égale à 0,013 .

La dégradation plus rapide de l'ARN $N_{I}$ lors du jeûne pourrait s'expliquer par la 
levée du mécanisme de rétro-contrôle entraînant une stimulation de la biosynthèse des ARN nucléaires (Quirin-Stricker et Mandel, 1968). Le maintien à un niveau constant du taux d'ARN ${ }_{n}$ lors de l'inanition prolongée de la carpe (Bouche, 1975) malgré une augmentation des échanges noyau $\rightarrow$ cytoplasme, pourrait être assuré par un accroissement complémentaire des synthèses. L'intensification de la sécrétion d'hormone somatotrope (S.T.H.) lors du jeûne total de la carpe (Olivereau, 1970) confirmerait cette hypothèse si le mode d'action de cette stimuline sur le métabolisme nucléique de l'hépatocyte de ce cyprinidé est le même que celui décrit par Salaman, Betteridge et Korner (1972) dans le noyau des cellules hépatiques du rat.

Ce travail constitue une étape dans l'étude des mécanismes adaptatifs assurant la longue survie des Poissons lors de l'inanition prolongée.

\section{Conclusion.}

Les temps de renouvellement des ARN total, ribosomal et polysomal du foie de carpes régulièrement nourries sont plus longs que ceux trouvés chez les Mammifères.

Le jeûne total et provoqué entraîne une augmentation de la vitesse de renouvellement de l'ARN total et de l'ARN ${ }_{r}$ de l'hépatocyte. Le maintien à des niveaux élevés des taux fractionnels de catabolisme et de synthèse après un jeûne de longue durée montre que l'ARN total ef l'ARN $N_{r}$ qui persistent en fin d'inanition conservent une activité métabolique élevée.

Les augmentations de la vitesse de renouvellement des ARN cytoplasmiques $\left(A R N_{r}\right.$ essentiellement) sont synchrones d'une accélération des exportations de l'ARN $N_{n}$ vers le cytoplasme. Des modifications de même nature ont été signalées chez les Mammifères soumis au jeûne protéique.

Accepté en juin 1976.

\section{Références}

BLOBEL G., POTTER V. R., 1968. Distribution of radioactivity between the acid-soluble pool and the pools of RNA in the nuclear non sedimentable and ribosome fractions of rat liver after a single injection of labeled orotic acid. Biochim. biophys. Acta, Pays-Bas, 166, 48-57.

BOUCHE G., GREACH Y., GAS N., 1970. Le jeône et la réalimentation chez la carpe (Cyprinus carpio L.). I. Influence sur les acides nucléiques du foie. Arch. Sci. Physiol., Fr., 24, 243-251.

BOUCHE G., NARBONNE J. F., CREACH Y., 1972. Le jeûne ef la réalimentation chez la carpe (Cyprinus carpio L.). VI. Influence sur les polysomes de l'hépatocyte. Arch. Sci. Physiol., Fr., 26, 111-120.

BOUCHE G., NARBONNE J. F., SERFATY A., 1972. Le jeône et la réalimentation chez la Carpe (Cyprinus carpio L.). III. - Influence sur l'ARN polysonal et ribosomal et sur l'ARN soluble. Arch. Sci Physiol., Fr., 26, 101-109.

BOUCHE G., 1975. Recherches sur les acides nucléiques et la protéosynthèse lors du jeûne prolongé et la réalimentation chez la carpe. Thèse Doct. Sci., Toulouse.

BOUCHE G., VELLAS F., 1975. Les vitesses de renouvellement des protéines hépatiques musculaires ef plasmatiques de la carpe (Cyprinus carpio L.) soumise à un jeûne total et prolongé. Comp. Biochem. Physiol., 51 A, 185-193.

BURTON K., 1956. A study of the conditions and mechanism of the diphenylamine reaction for the colorimetric estimation of deoxyribonucleic acid. Biochem. J., 62, 315-323. 
BUSCH S.,WEILL J. D., LEDIG M., MANDEL P., 1958. Effet de la carence protéique sur les nucléosides polyphosphates et la biosynthèse de l'acide ribonucléique dans le liquide cytoplasmique du foie de Rat. Bull. Soc. Chim. biol., Fr., 40, 1487-1503.

CAMPBELL R. M., KOSTER.LITZ H.W., 1952. The effects of dietary protein fat and choline on the composition of the liver cell and the turnover of phospholipid and protein-bound phosphorus. Biochim. biophys, Acto, Poys-Bas, 8, 664-679.

CREACH Y., 1972. Le jeûne expérimental chez la carpe : métabolisme azoté et équilibre hydrominéral. Thèse Doct. Sci., Toulouse.

ENWONWU C. O., MUNRO H. N., 1970. Rate of RNA turnover in rat liver in relation to intake protein. Arch. Biochem. Biophys., U.S.A., 138, 532-539.

ENWONWU C. O., STAMBAUGH G., SREENBY L., 1971. Synthesis and degradation of liver ribosomal RNA in fed and fasted rats. J. Nutr., U.S.A., 101, 337-346.

FONTAINE M., OLIVEREAU M., 1963. Nutrition et sexualité chez les poissons. Journées scientifiques du Centre National de Coordination des Etudes ef Recherches sur la Nufrition ef I'Alimentation, 11, A $125-A 148$.

GERBER G., GERBER G., ALTMAN K. I., 1960. The catabolism of tissue nucleic acid in the rat. II. Turnover tissue of ribonucleic and free nucleotides. J. Biol. Chem., U.S.A., 235, 2682-2687.

HADJOLIOV A. A., 1966. Studies of the turnover and messinger activity of rat liver ribonucleic acids. Biochim. biophys. Acta, Pays-Bas, 119, 547-556.

HIRSCH C. A., HIATT H. H., 1966. Turnover of liver ribosomes in fed and in fasted rats. J. biol. Chem., U.S.A., 241, 5936-5940.

JARNUM S., JANSEN K. B., 1972. Plasma protein turnover (Albumin, transferrin, lgC, Igns) in Menetrier's disease (giant hypertrophic gastritis) : evidence of non-selective protein loss. Gut, G. B., 13, 128-137.

LOEB J. N., HOWELL R. R., TOMKINS G. M., 1965. Turnover of ribosomal RNA in rat liver. Science, U.S.A., 149, 1093-1095.

LOVE R. M., 1970. Chemical biology of fishes. Academic Press, London and New York.

MISHRA R. K., WHELDRAKE J. F., FELTHAM L. A. W., 1972. RNA turnover in endoplasmic reticulumbound and free ribosomes. Biochim. biophys. Acto, Pays-Bas, 281, 393-395.

MOULE Y., 1953. Etudes des acides nucléiques et de certains dérivés organiques ef inorganiques du phosphore dans les tissus animaux. Arch. Sci. Physiol., Fr., 7, 161-187.

OLIVEREAU M., 1970. Stimulation des cellules somatotropes de l'hypophyse de la carpe après un jeûne prolongé. C. R. Acod. Sci., Fr., 270, 2343-2346.

QUIRIN-STRICKER C., MANDEL P., 1968. Etude du renouvellement du RNA des polysomes, du RNA de transfert et du RNA « messager 》 dans le foie de rat soumis à un jeûne protéique. Bull. SOC. Chim. biol., Fr., 50, 31-45.

SALAMAN D. F., BETTERIDGE S., KORNER A., 1972. Early effects of growth hormone on nucleolar and nucleoplasmic RNA synthesis and polymerase activity in normal rat liver. Biochim. biophys. Acta, Pays-Bas, 272, 382-395.

SCHMIDT G., THANNHAUSER S. H., 1945. A method for the determination of deoxyribonucleic acid and phosphoproteins in animal tissues. J. Biol. Chem., U.S.A., 161, 83-89.

SORVACHEV K. F., 1957. Etude électrophorétique des fractions protéiques du sérum de carpes d'étang élevées dans diverses conditions (en Russe). Zool. Zh. S.S.S.R., 36, 737-741.

VANNEMAKER R.W., BANKS W. L., WUNNER W. H., 1965. Use of a single tissue extract of determine cellular protein and nucleic acid concentration and rate of amino acid incorporation. Anal. Biochem., U.S.A., 11, 320-334.

WEBER G., SINGHAL R. L., SRIVASTAVA S. K., 1965. Effect of nutritional state on hormonal regulation of liver enzymes. Canad. J. Biochem., 43, 1549-1563. 